\title{
Existe evidencia para respaldar intervenciones paliativas y cuidados durante el fin de la vida
}

There is evidence to support paliative interventions and care during the end of of life

\section{Objetivo}

Evaluar la evidencia disponible sobre intervenciones paliativas y cuidados durante el fin de vida (CFV).

\section{Selección de estudios}

Búsqueda en MEDLINE desde 1990 hasta 2004 y en resúmenes y revisiones del Consenso Nacional en Calidad en Medicina Paliativa de EEUU (2007) con supervisión de la Academia Americana de Hospices y Medicina Paliativa. Fue evaluada la mejor evidencia para cada tópico priorizando los estudios de intervención (EI) las revisiones sistemáticas (RS). Fueron usadas las palabras clave "fin de vida", "enfermedad terminal, avanzada, crónica y/o fatal", "mal pronostico", "insuficiencia cardiaca crónica" (ICC) "síndrome de inmunodeficiencia adquirida" (SIDA) "diálisis", "enfermedad pulmonar obstructiva crónica" (EPOC) "demencia" y "cáncer".

La búsqueda se orientó a los tópicos descriptos en el cuadro 1.

Cuadro 1: tópicos a los que se orientó la búsqueda de la revisión sistemática de Lorenz y col.

1) Las características comunes durante el final de la vida (bienestar, prevención y control de síntomas, toma de decisiones, información, bienestar emocional-espiritual, funcionalidad y sobrevida);

2) La identificacion de los pacientes que se beneficiarían de la
Lorenz K y col. Ann Intern Med. 2008; 148:147-159. intervención (estado funcional, riesgo de complicaciones, gravedad de su condición clínica);

3) Las estrategias terapéuticas utilizadas para el dolor, la disnea y la depresión (ej. fármacos y terapias alternativas);

4) La planificación anticipada de los cuidados (muerte en el domicilio, decisiones subrogadas, estadía en geriátricos, "hospices", etc.);

5) Las oportunidades efectivas para mejorar los CFV (seguimiento multidisciplinario, continuidad, seguimiento telefónico, hospitalización, cuidados en domicilio, directivas anticipadas);

6) La evaluación de la efectividad de los CFV y de la satisfacción quienes los prestan y los reciben (prestadores de salud y familiares).

Cada trabajo fue calificado de acuerdo a su diseño, su calidad y a la consistencia de sus resultados para cada área. A través del método de GRADE*, se clasificó a la evidencia sobre cada área como alta, moderada, baja o muy baja.

\section{Resultados principales}

De un total de 24423 estudios encontrados, 6381 fueron considerados relevantes y 1274 sometidos a una revisión minuciosa. En la tabla 1 se resumen los resultados de 33 revisiones sistemáticas y 89 estudios de intervención sobre dolor, disnea, depresión, planificación anticipada de los CVF, continuidad y cuidadores.

Tabla 1: nivel de evidencia que avala a las diferentes intervenciones paliativas y cuidados durante el fin de la vida

\begin{tabular}{|c|c|c|c|c|}
\hline & RS & EI & Evidencia & Intervenciones \\
\hline \multirow[t]{3}{*}{ Dolor } & \multirow[t]{3}{*}{9} & \multirow[t]{3}{*}{24} & Fuerte & $\begin{array}{l}\text { AINE, opioides, radionucleidos, radioterapia, bifosfonatos para dolor por metástasis ós eas (mama, mieloma } \\
\text { múltiple). }\end{array}$ \\
\hline & & & \multirow[t]{2}{*}{ Baja } & Equipo multidisciplinario \\
\hline & & & & Bifosfonatos par el dolor por fracturas \\
\hline \multirow[t]{2}{*}{ Disnea } & \multirow[t]{2}{*}{2} & \multirow[t]{2}{*}{12} & Fuerte & B-agonistas, opioides; rehabilitación respiratoria y oxigenoterapia (EPOC); opioides (disnea por cáncer). \\
\hline & & & Baja & Oxigenoterapa en disnea (cáncer) \\
\hline Depresión & 4 & 9 & Fuerte & $\begin{array}{l}\text { Psicoterapia, intervenciones psicosociales cognitivas y/o no cognitivas, inhubidores de recaptación de } \\
\text { serotonina y tricclicos (cáncer). }\end{array}$ \\
\hline $\begin{array}{l}\text { Planificación anticipada de } \\
\text { los cuidados }\end{array}$ & 9 & 32 & \multirow[t]{3}{*}{ Moderada } & Intervenciones multifactoriales incrementan las directivas anticipadas. \\
\hline Continuidad de los cuidados & 9 & 12 & & Intervenciones multidisciplinarias para reducir re-internaciones (ICC). \\
\hline \multirow[t]{2}{*}{ Agotamiento de los cuidadores } & \multirow[t]{2}{*}{8} & \multirow[t]{2}{*}{19} & & $\begin{array}{l}\text { Intervenciones para mejorar el grado de satisfacción del cuidador (demencia); y para mejorar la comunicación } \\
\text { y el proceso de duelo. }\end{array}$ \\
\hline & & & Baja & Intervenciones para mejorar el grado de satisfacción del cuidador (cáncer). \\
\hline
\end{tabular}

RS: revisiones sistemáticas. El: estudios de intervención.

\section{Conclusiones}

Existe fuerte evidencia para respaldar el uso de intervenciones paliativas para el control del dolor, la disnea y la depresión. Por otro lado existe evidencia de que las intervenciones multifactoriales y contínuas facilitan las directivas anticipadas, mejoran la satisfacción de los cuidadores de pacientes con demencia y se asocian a una disminución del número de internaciones por ICC.

Palabras claves: cuidados paliativos, revisión sistemática, eficacia.

Keywords: palliative care, systematic review, efficacy.

Fuentes de financiamiento: National Institute of Nursing Research, Agency for Development Service Career Development Award, National Cancer Institute.

Gloria Vega [ Medica de Familia, Unidad de Cuidados Paliativos del Hospital Enrique Tornú, Buenos Aires, Argentina. ]

Sergio Terrasa [ Servicio de Medicina Familiar y Comunitaria del Hospital Italiano de Buenos Aires, gloriafanny@gmail.com. ]

Recibido el 15/02/09 y aceptado el 31/03/09.

Vega G y Terrasa S. Existe evidencia para respaldar intervenciones paliativas y cuidados durante el fin de la vida. Intervenciones basadas en la evidencia para mejorar los cuidados paliativos del dolor, la disnea y la depresión en el final de la vida. Evid. Act. Pract. Ambul. 12(2). 50. Abr-Jun. 2009 Comentado de: Lorenz K y col. Evidence for improving Palliative Care at the End of life: a Sistematic Review. Ann Intern Med. 2008; 148:147-159. 